\title{
Energy Efficient and Subcarrier Allocation for OFDMA based Cooperative Relay Networks
}

\author{
Vijayabharathi.G \\ M.Tech , Final Year \\ Department of Electronics and \\ Communication Engineering \\ Sri Manakula Vinayagar \\ Engineering College, \\ Madagadipet, Puducherry-605017
}

\author{
Vijayalakshmi.A \\ Associate Professor \\ Department of Electronics and \\ Communication Engineering \\ Sri Manakula Vinayagar \\ Engineering College, \\ Madagadipet, Puducherry-605017
}

\begin{abstract}
Energy efficient wireless communication is important for battery constrained mobile devices. For mobile devices in a cellular system, uplink power consumption dominates the wireless power budget because of RF power requirements for reliable transmission over long distances. In this work we developed a low-complexity subcarrier-power allocation and relay selection scheme has been developed for maximizing energy efficiency per user in an Orthogonal Frequency Division Multiple Access (OFDMA) cooperative relay network, using full channel state information (CSI), which is available at both relay and BS nodes. The previous works in this area focused on optimizing energy efficiency by maximizing the instantaneous bits-per-Joule metric through Equal-Power Allocation (EPA) schemes resulted in significant energy savings for uplink cellular OFDMA transmissions. It also shows that the proposed scheme has significant improvement on the energy efficiency performance as compared with EPA schemes which allocate equal transmit power to the assigned subcarriers. The simulation results show that the proposed scheme can achieve better performance with less computational complexity as compared to the exhaustive search scheme.
\end{abstract}

\section{Keywords}

Energy efficiency, OFDMA, bits per Joule, Equal power control, channel state information.

\section{INTRODUCTION \\ 1.1 OFDMA}

It is a special kind of FDM. The spacing between carriers is such that they are orthogonal to one another. Therefore no need of guard band between carriers. Orthogonal frequencydivision multiple access (OFDMA) is the chosen multicarrier technique for the current and future cellular systems such as mobile Worldwide Interoperability of Microwave Access (WiMAX; IEEE 802.16e) and Third-Generation Partnership Project Long-Term Evolution (3GPP LTE).

Each base station will be responsible for its own user's resources, and there should be minimum interactions between neighbours BSs. In such systems, the information from the source to the destination is not only transmitted via a direct link but also forwarded via relays. Power efficiency is becoming increasingly important for wireless communication systems due to limited battery resources in mobile devices. Hence, recent energy-efficient management schemes have focused on minimizing energy consumption rather than throughput maximization. Additionally, orthogonal frequency division multiple access has emerged as one of the prime multiple access schemes for next generation multi-user broadband wireless networks. However, limited research has been done for energy-efficient communication in OFDMA systems. Besides the substantial studies of power allocation schemes for relay networks, energy-efficient communication has also been investigated. Bits-per-energy efficiency has been studied. An energy-efficient design for maximizing 'bits per joule' for a single BS system was investigated. A gametheoretic approach for maximizing energy efficiency, defined as 'bits per energy', in a user-cooperation network where one MS can transmit data collaboratively via another MS was proposed. A relay selection scheme for energy saving was proposed for a cooperative relay system where transmit data is beamformed by a set of relays.

Significant amount of research on cooperative communication techniques has been done to allow stations to cooperate in transmissions to improve the overall performance of the network. In this work, we studied the joint power allocation for RS and MS and the relay selection problem under the constraint of limited RS transmit power in order to maximize the energy efficiency per user defined as 'bits per joule' for uplink transmission in an OFDMA cooperative relay network.

\subsection{Cooperative Relay Network}

We considered the scenario depicted in Figure 1, which includes a single relay with source and destination nodes. Cooperative relaying schemes suffer from the "orthogonality constraint", for the assignment of orthogonal resources for reception and transmission at the relay. Without loss of generality, we focused on the time division case and divide the available channel into two orthogonal sub channels in the time domain. In order to achieve the same end-to-end spectral efficiency, we need to double the spectral efficiency on each of the individual links.The source sends a broadcast message to destination and relay. The relay then forwards additional information about the source message to the destination, which appropriately combines the received data.

The rest of the paper is organized as follows. Section 2, represents the system model and a class of cooperation protocols for multinode wireless networks. Section 3 describes the relay selection process. Section 4, explains the 
energy efficient power allocation. Section 5, gives the performance analysis of the proposed energy efficient scheme. Section 6 concludes this paper.

\section{SYSTEM MODEL}

We consider A multi-user OFDMA relay network with Ksubcarriers denoted by $\hat{\mathrm{E}}=\{1,2,3, \ldots, \mathrm{K}\}$, a single Base Station (BS) node r1, Relay (RS) nodes $\gamma=\left\{r_{i} \mid \mathrm{i}=2,3, \ldots, \mathrm{J}+1\right\}$, and Mobile Station (MS) nodes $\mathrm{B}=\left\{n_{i} \mid \mathrm{i}=1, \ldots, \mathrm{N}\right\}$, randomly distributed within the cell. We assume that the uplink channel state information is perfectly known at both BS node and relay nodes, and only the Decode-and-Forward (DF) cooperative relay is considered. Orthogonal transmissions are used for simultaneous transmissions among different source nodes by using different channels, e.g., different frequency bands and time division duplex (TDD) is employed by DF relay nodes for each user. The Maximum-Ratio-Combining (MRC) detector is employed by the BS node, assuming perfect decoding at the relays. Similar to the conventional relay-based cooperative communication systems, we assume a two-time-slot transmission scheme with perfect timing synchronization among all users within the cell. In the first time slot, MSs transmit data to BS or relay nodes while BS and relay nodes receive data. In the second time slot, relay nodes transmit data received from MSs in the first time slot to BS while MS keeps transmitting data to BS via direct link without relaying. Each subcarrier suffers from frequencyselective block fading, i.e. the channel state remains the same within each frame, which means two-time-slot here. Data streams transmitted by MSs or relay nodes would be carried by same carriers in the two-time-slot transmission.

However, this work also focused on a single sourcedestination transmission and thus multi-user interference is negligible. Alternatively, multi-access relay techniques deal with many sources which share the same relay in order to deliver their data to a single destination. The proposed relay selection criteria lack the flexibility to deal with the presence or absence of interference effects and remain optimal for ideal scenarios without interference. To develop a low-complexity subcarrier-power allocation and relay selection scheme for maximizing energy efficiency per user in an OFDMA cooperative relay network

\section{RELAY SELECTION CRITERIA}

Amplify-and-Forward protocols are of interest since the relay nodes simply retransmit an amplified version of the received signal and thus have a low complexity. We propose a class of cooperative decode-and-forward protocols for arbitrary $\mathrm{N}$ relay wireless networks, in which each relay can combine the signal received from the source along with one or more of the signals transmitted by previous relays.

We consider selective relaying in which each receiving relay can judge on the quality of the receiving signal and decide whether to forward the received signal or not. This can be implemented in a practical system by allowing each relay to measure the received signal-to-noise ratio (SNR) and forward the signal if the SNR is higher than the prespecified threshold. Another possibility is to employ cyclic-redundancy-check encoding to data frames. In order to verify the effectiveness of the proposed scheme, we compare the performance of the proposed scheme with that of other scheme, i.e., EPA each subcarrier must be assigned to only one MS during a twotime-slot period to avoid inter-subcarrier interference. The subcarrier assigned to relay node should be the same as the subcarrier assigned to MS during a two-time-slot period.
Current and evolving standards for broadband wireless systems (e.g., IEEE 802.16, 3G LTE, LTE Advanced, etc.) are adopting orthogonal-frequency division multiple access (OFDMA) as the resource allocation policy, in which the available time and frequency resources over each wireless link are orthogonally allocated across users, avoiding interuser interference and impairments due to multipath fading. An intrinsic advantage of OFDMA over other multiple access methods is its flexibility; which allows it to realize multiuser diversity gains over both time and frequency domains through scheduling mechanisms. To further maximize spectral efficiency, it is also preferable to reuse spectrum opportunistically under OFDMA resource allocation; in fact future cellular networks are evolving toward frequency reuse of one, i.e. all cells/sectors operate on the same frequency channel.

\section{PROPOSED ENERGY EFFICIENT POWER ALLOCATION}

\subsection{Energy Efficiency Metrics}

In this section, we proposed a low-complexity Maximum Energy-Efficiency (MaxEE) scheme to solve the above problem. In comparison with the greedy algorithm accomplished by the exhaustive search, the complexity of our scheme is significantly reduced. Energy efficiency has been defined in several ways. One way is to take the ratio of the transmitted bit rate per unit time to the transmitted power (plus circuit power), measured in bits/Joule. The objective is to increase the number of transmitted information bits per unit energy which has been widely used. The definition for EE might lead to false conclusions especially at low power scenarios due to the lack of linearity between the consumed energy and transmitted power. A different way to define the $\mathrm{EE}$ is to use the energy consumption per bit, i.e., the ratio of consumed power per achievable rate as a metric measured in Joules/bit. The energy consumption increases linearly with transmitted power. The objective here is to minimize the total energy consumption for a given data rate.

\subsection{Bits-Per-Energy Efficiency}

Bits-per-energy efficiency is an elegant information-theoretic characterization for the single-user, multiple-access, and interference channels. The energy efficiency of the relay channel is studied. However, maximizing energy efficiency almost invariably drives users to the low SNR regime, where rates are arbitrarily small. Strict maximization of energy efficiency, then, is not typically useful in practice when users have target rates to meet or delay constraints to satisfy.

First, we define the bits-per-energy efficiency. Since each time block has the same duration, the amount of data having reached the base station is proportional to the sum of rates over all previous time blocks. Similarly, the total energy expenditure is proportional to the sum of the power allocations in previous time blocks. So, the total bits-perenergy efficiency for each user is proportional to where the sums converge to expectations because the channels are ergodic, allowing us to write the power allocations as functions of the channel coefficients, denoted by the vector $\mathrm{h}$. The units of $e_{1}$ and $e_{2}$ are normalized bits per joule: if each users' transmitter power were 1 Watt and each channel were 1 $\mathrm{Hz}$ wide, the units of $e_{1}$ and $e_{2}$ would be bits per joule; we simply refer to the units as "bits per energy".

$e_{1}=\frac{E_{h}\left[r_{1}\right]}{E_{h}\left[p_{s 1}(h)+p_{r 1}(h)\right]}$ 
$e_{2}=\frac{E_{h}\left[r_{2}\right]}{E_{h}\left[p_{s 2}(h)+p_{r 2}(h)\right]}$

$e_{i}\left(p_{r 1}, p_{r 2}\right)=\frac{E_{h}\left[r_{i}\left(p_{r j}(h)\right)\right]}{1+E_{h}\left(p_{r j}(h)\right)}$

Now, to sidestep the obstacle of users' rates becoming small, we constrain each user to employ full power for its own transmission. That is, we always have $p_{s i}(t)=1$, meaning that each user's rate is at least as high as the capacity of its link with the BS. In addition to bounding users' rates away from zero, this restriction approximates a cellular environment, where users simply use the available power to achieve the highest possible quality of service (neglecting, perhaps, the need for power control).

The bits-per-energy region is entirely characterized by the channel statistics. $\varepsilon$ is necessarily compact, but it need not be convex, since time-sharing between power allocations does not result in convex combinations of bits-per energy efficiencies. Each point in $\varepsilon$ is achieved by using a different relay power allocation pair $p_{r 1}(h), p_{r 2}(h)$. While the set of feasible power allocations is infinite-dimensional, we can characterize the power allocations associated with the outer boundary of $\varepsilon$.

$\varepsilon=\left\{\begin{array}{c}\left(e_{1}\left(p_{r 1}, p_{r 2}\right), e_{2}\left(p_{r 1}, p_{r 2}\right)\right): \\ 0 \leq p_{r 1}(h), p_{r 2}(h) \leq 1\end{array}\right\}$

\subsection{Optimum Power Allocation}

The proposed relay selection scheme is able to extract the full diversity. However, further performance improvement over EPA is possible through optimum power allocation (OPA). In the following, we pursue two directions for performance optimization. We assume that power is divided between source and the selected relay node irrespective of relay location. In this case, power of the source and relay node are given by $K_{S} P$ and $\left(1-K_{S}\right) \mathrm{P}$ respectively. The optimization problem can be formulated as

$\min _{\text {s.t. }} \quad 0<K_{S<1} P_{e}\left(K_{S}\right)$

$\min _{\text {s.t } K_{S}+\sum_{i=1}^{N} P_{i K_{i}=1}} P_{e}\left(K_{S}, K_{1}, K_{2} \ldots \ldots, K_{N}\right)$

The optimized power allocation has significant effect on the error rate performance of cooperative systems. By distributing the available power to the transmitting nodes based on their respective locations, not only we have performance improvement, but also we can reduce unnecessary interference created by transmitting nodes to co-existing wireless systems. We assume that power is divided among source and relay nodes taking into account location of relay nodes. Power of the source is $K_{S} P$ and power of each relay node is $K_{i} P, \mathrm{i}=1,2, \ldots, \mathrm{N}$ such that $K_{S}+\sum_{i=1}^{N} P_{i K_{i}=1}$. Recall that $P_{i}$ has been earlier defined and denotes the probability of $i^{\text {th }}$ relay node being selected. The optimization problem now takes the form of analytical solutions for (5) and (6) are unfortunately very difficult to obtain, if not infeasible. It should be also noted that this optimization problem needs not to be solved in real-time for practical systems, because the optimization does not depend on the instantaneous channel information or the input data.

\section{PERFORMANCE ANALYSIS}

we consider an OFDMA two-hop relay network with a certain number of MSs uniformly distributed within a 600-meter radius cell having 6 relay nodes deployed with equal angle spacing on a circle with 300 -meter radius centred at the BS. Each transmitted signals experience path-loss proportional to $d^{-2}$, where $d$ denotes the distance between the transmitting MS and the receiving node, and each subcarrier suffers from i.i.d. Rayleigh fading with unity variance. The AWGN variance is assumed to be $1.4 \times 10^{-5}$.

Table 1. Simulation Parameters

\begin{tabular}{|c|c|}
\hline PARAMETER & VALUE \\
\hline Number of Carrier & 32 \\
\hline Number of Relay & 6 \\
\hline Circuit power & $12 \mathrm{dBm}$ \\
\hline
\end{tabular}

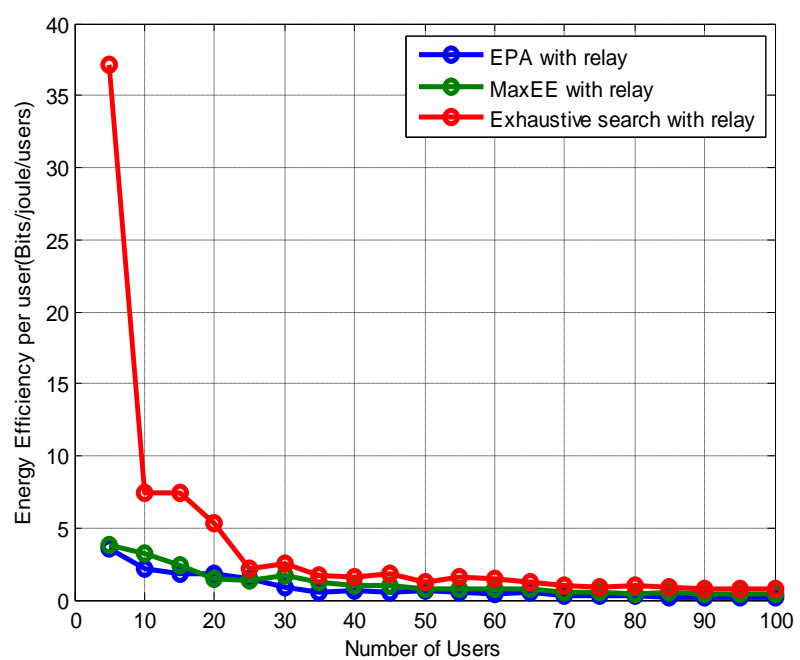

Fig 1 : energy efficiency for users

Fig 1 evaluates energy efficiency for users. The proposed MaxEE scheme achieves the highest energy efficiency for users compared with EPA scheme, which demonstrates the spectrum efficiency of the proposed MaxEE scheme outperforms that of the other scheme. The proposed MaxEE scheme and the exhaustive search achieve the best energy efficiency performance shown in fig 4.1.The Performance of energy efficiency per user for different number of relay nodes deployed in the cell. The energy-efficient subcarrier-power allocation and relay selection scheme in multi-user OFDMA cooperative relay networks. To maximize average transmitting bits per joule for uplink transmission. Firstly, we express average bit rate per watts as the ratio of the average achievable rate to the average consumed power for MS. The energy efficiency degrades with the decrease of the number of relay nodes deployed in the cell since the opportunity for a MS to transmit via relay link is reduced. 


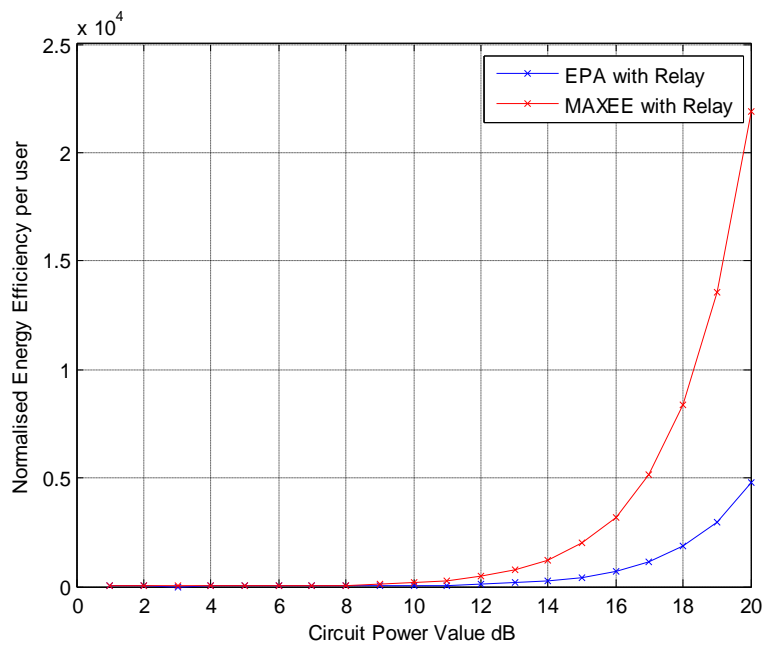

Fig 2: normalised energy efficiency vs circuit power

Fig.2 illustrates MaxEE scheme obtains very similar performance with a much-lower complexity as compared to the exhaustive search. In addition, the proposed scheme outperforms the two conventional EPA schemes over $60 \%$ since they do not consider the user's energy efficiency. The outcome of normalizing the original result by the best one, the performance of energy efficiency for different MS circuit power is revealed. It is shown that the gain of the proposed algorithm decreases with the increase of the MS circuit power. It means the proposed scheme is more effective for devices with low-power consumption.

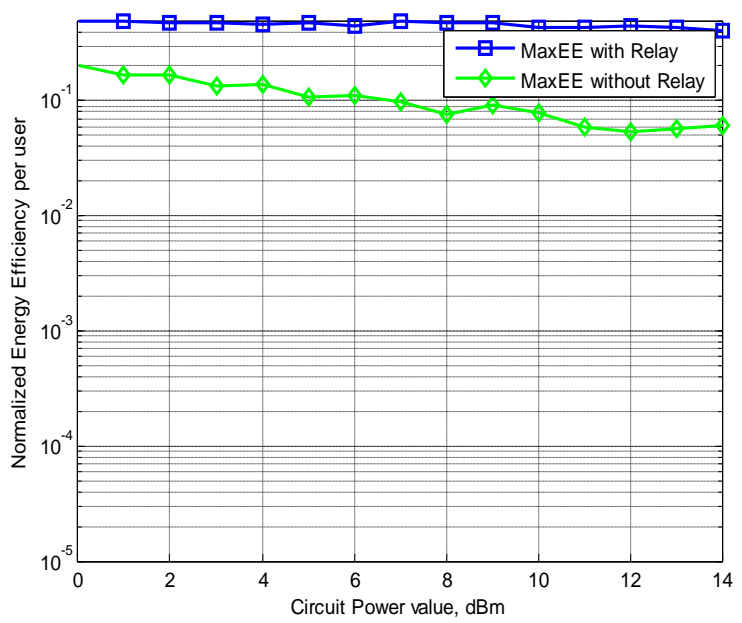

Fig 3: relay schemes of MaxEE

The MaxEE without relay scheme applies the proposed MaxEE scheme for a single BS system with no relay nodes deployed in the cell, and it has similar performance as the scheme proposed. The MaxEE without relay scheme, other scheme is simulated in a single cell with relay nodes deployed shown in fig 3.

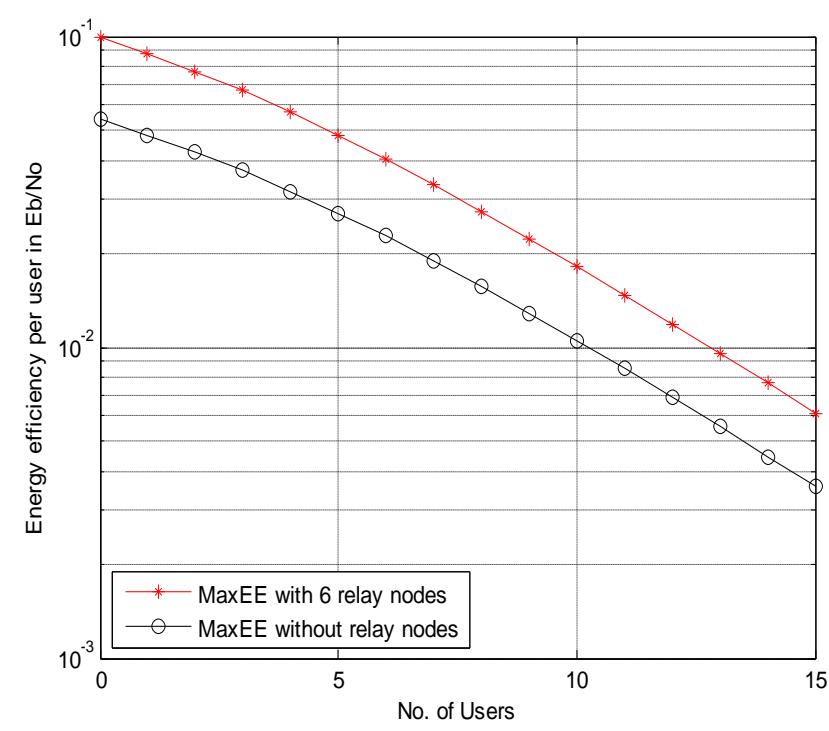

Fig 4: energy efficiency in $e_{b} / n_{0}$ for users

The noise spectral density $\mathrm{N}_{0}$, usually expressed in units of watts per hertz, can also be seen as having dimensions of energy, or units of joules, or joules per cycle. $\mathrm{E}_{\mathrm{b}} / \mathrm{N}_{0}$ is therefore a non-dimensional ratio. Fig 4 illustrates $E_{b} / N_{0}$ is commonly used with modulation and coding designed for noise-limited rather than interference-limited communication, since additive white noise with constant noise density $\mathrm{N}_{0}$ is assumed.

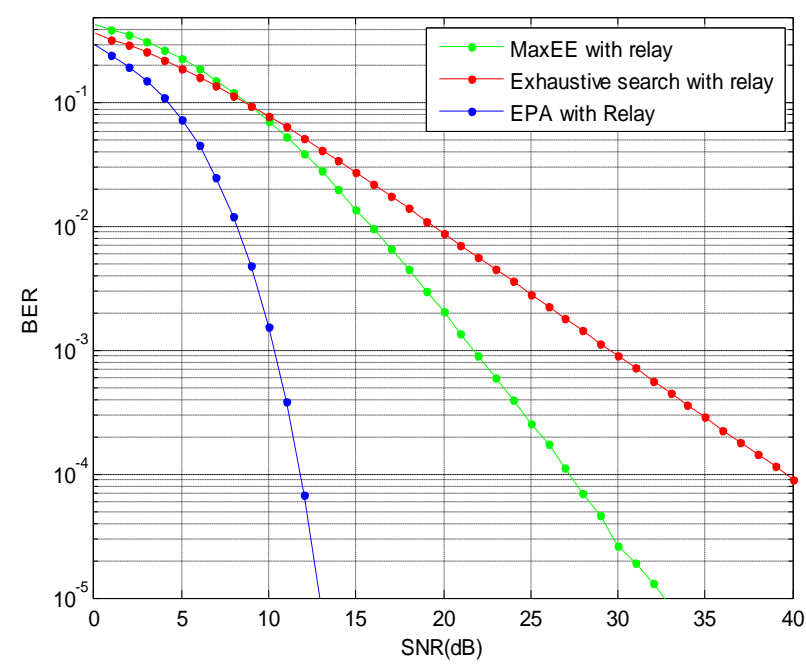

Fig 5: BER vs SNR

Fig 5 shows that the proposed low complexity scheme is referred to the Maximum-Energy-Efficiency scheme, and the exhaustive search scheme chooses the optimal assignment combination for subcarrier-power allocation which achieves maximum energy efficiency in each transmission period. The Equal Power Allocation for max-throughput scheme allocates equal transmit power for each subcarrier assigned to a MS having the maximum rate. The Equal Power Allocation for fairness scheme has two steps for subcarrier-power allocation. In the first step, subcarriers would be allocated equal transmit power and assigned to each MS until every MS' minimum data rate has been satisfied. In the second step, the rest of 
unassigned subcarriers would be allocated with equal transmit power and assigned to the MSs having the maximum rate.

\section{CONCLUSION}

In this paper, we have developed a low-complexity subcarrierpower allocation and relay selection scheme for maximizing energy efficiency per user in an OFDMA cooperative relay network. We consider a multi-user OFDMA DF relay network, and utilize the additional transmission opportunity provided by cooperative relaying with Maximum Ratio Combining (MRC) to further improve the energy efficiency of the network. In order to maximize the energy efficiency for the relay link, the optimal joint power allocation for MS and RS is derived. Based on that, we proposed the Max-EnergyEfficiency (MaxEE) scheme which jointly performs subcarrier allocation, MS-RS joint power allocation, and RS selection, and achieves very similar performance in much lower complexity as compared to the exhaustive search.

As future work focusing on the problem of designing efficient resource allocation schemes for an OFDMA-based multiuser cooperative communication system that uses amplify and forward relaying. Using a two-phase relaying protocol, we assume that both the source and the relay have fixed power constraints and that the source employs a selective relaying mechanism. The first part is to sub optimally allocate subcarriers to different users based on proportional rate fairness. The next part is a two step iterative approach to find relay decisions and power allocation at the source during the first phase and at the relay during the second phase. The last part is a water-filling algorithm to allocate power at the source during the second phase to all non relaying subcarriers

\section{REFERENCES}

[1] Wenbing Dang, Jianwei Huang, "Subcarrier-pair based resource allocation for cooperative $\mathrm{AF}$ multi-relay OFDM systems", IEEE transactions on wireless communications, vol. 9, no. 5 ,may 2010.

[2] YinRui ,Zhang Yu, Zhang Jietao Yu, Guanding and Zhaoyang," Joint resource allocation in multiple channels,multiple relays systems",IEEE transactions on wireless communications and networking conference, april 2010.

[3] Y. Chen and S. Kishnore, "A game-theoretic analysis of decode and-forward user cooperation," IEEE trans. wireless communications, vol. 7, no. 5, pp. 1941-1951, may 2008 .
[4] A. Host-Madsen and J. Zhang, "Capacity bounds and power allocation for wireless relay channels," Information Theory, IEEE transactions on, vol. 51, no. 6, pp. 2020-2040, june 2005.

[5] L.Vandendorpe, J. Louveaux, O. Oguz and A. Zaidi, "Rate-optimized power allocation for DF-relayed OFDM transmission under sum and individual power constraints,"EURASIP journal on wireless communications and networking, vol. 2009, article id 814278, 2009.

[6] R. Madan, N. B. Mehta, A. F. Molisch, and J. Zhang, "Energy-efficient cooperative relaying over fading channels with simple relay selection", IEEE trans. wireless commun., vol. 7, no. 8, pp. 3013-3025, aug. 2008.

[7] Y. Li, B. Vucetic, Z. Zhou, and M. Dohler, "Distributed adaptive power allocation for wireless relay networks," IEEE trans.wireless commun., vol. 6, pp. 948-958,mar. 2007.

[8] L. Vandendorpe, R. T. Duran, J. Louveaux, and A.Zaidi, "Power allocation for OFDM transmission with DF relaying," in proc. IEEE int. conf. commun. (icc), pp. 3795-3800, may 2008.

[9] Khoa T. Phan, Long Bao Le, Sergiy A.Vorobyov, and Tho Le-Ngoc, "Centralized and distributed power allocation in multi-user wireless relay networks," in proc. IEEE int. conf. commun. (ICC), may 2009.

[10] S. H. Nam, M. Vu, and V. Tarokh, "Relay selection methods for wireless cooperative communications," in proc. conf. on info.sciences and systems (CISS), 2008.

[11] Khoa T. Phan, Duy H. N. Nguyen, and Tho Le-Ngoc, "Joint power allocation and relay selection in cooperative networks,"in proc.IEEE global telecommun. conf. (GLOBECOM), 2009.

[12] A.Sendonaris, E. Erkip, and B. Aazhang, "User cooperation diversity, part I: system description," IEEE trans. commun., vol. 51, no. 11, pp.1927-1938, nov. 2003.

[13] G. Miao, N. Himayat, Y. Li, and D. Bormann, "Energyefficient design in wireless OFDMA," in Proc. ICC 2008,2008

[14] A. Bletsas, A. Khisti, D.P. Reed, A. Lippman, "A simple cooperative.diversity method based on network path selection" IEEE journal on selected areas in communications, vol. 24, no. 3, pp.659-672, mar.2006 